A group of mice were treated with either $33 \mathrm{mg} / \mathrm{kg}$ or $100 \mathrm{mg} / \mathrm{kg}$ imatinib (which produced blood levels similar to a mid-range dose in humans), administered 1 day before $\mathrm{CIA}$ induction. A further group of mice were treated with either $33 \mathrm{mg} / \mathrm{kg}$ or $100 \mathrm{mg} / \mathrm{kg}$ imatinib after established CIA had developed. Imatinib was an effective treatment for both established and new-onset CIA: in both experiments, imatinib-treated mice experienced lesssevere CIA symptoms and also showed fewer histologically visible signs of inflammation and tissue destruction than control mice (which did not receive imatinib).

In vitro studies in human and murine cell lines demonstrated that imatinib selectively inhibited multiple signal-transduction pathways that are known to be important in the pathogenesis of RA. In particular, imatinib inhibited plateletderived growth factor signaling in fibroblast-like synoviocytes from RA patients, and inhibited production of proinflammatory cytokines by several cell types. Imatinib also reduced B-cell proliferative responses to lipopolysacchide.

The authors conclude that imatinib might be a useful treatment for the autoimmune or inflammatory diseases characterized by fibroproliferation, and suggest prospective clinical trials to determine whether the results found in the CIA model are applicable to human RA.

Original article Paniagua RT et al. (2006) Selective tyrosine kinase inhibition by imatinib mesylate for the treatment of autoimmune arthritis. J Clin Invest 116: 2633-2642

\section{Inability to eliminate self-reactive and polyreactive immature B cells is a feature of SLE}

In patients with systemic lupus erythematosus (SLE), immature B cells that recognize self antigens fail to undergo apoptosis or suppression before maturation; one consequence is that untreated patients accumulate mature B cells capable of autoantibody production. Yurasov and colleagues suggest that the existence of such B cells predisposes patients with SLE to develop autoantibodies.

The authors obtained mature naive $B$ cells from six SLE patients aged 7-17 years (three provided samples during active disease and during sustained remission, and three provided samples during remission only). The authors cloned the antibodies expressed by these
B cells, and compared them with cloned antibodies from three healthy individuals. Analysis of cloned antibodies from SLE patients with active disease and in remission revealed abnormalities (with no consistent pattern) in immunoglobulin heavy and light chain recombination. Antibodies from SLE patients with active disease tended to have more reactivity to self antigens and more polyreactivity (another consequence of impaired elimination of undesirable B cells) than antibodies from patients in remission; however, antibodies from SLE patients in remission still had more self-reactivity $(P=0.025)$ and polyreactivity $(P=0.009)$ than those from healthy individuals.

The authors conclude that antibodies produced by mature, naive B cells from patients with SLE show considerable interindividual variation in self-reactivity and polyreactivity; these abnormalities persist irrespective of disease activity levels. They suggest that impaired elimination of self-reactive and polyreactive immature B cells is an underlying feature of SLE.

Original article Yurasov S et al. (2006) Persistent expression of autoantibodies in SLE patients in remission. J Exp Med 203: 2255-2261

\section{High-sensitivity C-reactive protein test results predict fracture risk in elderly women}

C-reactive protein (CRP) is an acute-phase reactant that is frequently used as a systemic marker of inflammation. Since proinflammatory cytokines stimulate osteoclastic bone resorption and inhibit osteoblast functions, Pasco et al. hypothesized that CRP levels in elderly women might predict their risk of fracture.

The authors recruited 444 women who participated in the Geelong Osteoporosis Study. Participants were aged $\geq 65$ years and had not used hormone-replacement therapy or glucocorticoids in the previous 6 months. Participants' serum CRP levels (measured by high-sensitivity CRP [hsCRP] assay), bone mineral density, markers of bone turnover, comorbidities (including prevalent fracture), medications, diet and lifestyle were assessed at enrollment. The women were followed up for a median of 5.5 years, until incident fracture (102 women), death (46 women), they moved away (13 women), or the end of the study. The median 